\title{
Motor Time of the Rectus Femoris under Loading of Different Weights
}

\author{
Ichiro Tsuji, Hiroshi Nagasaki and Ryuichi Nakamura \\ Institute of Rehabilitation Medicine, Tohoku University \\ School of Medicine, Narugo, Miyagi 989-68
}

Short Report

Tsuji, I., Nagasaki, H. and Nakamura, R. Motor Time of the Rectus Femoris under Loading of Different Weights. Tohoku J. exp. Med., 1985, 146 (1), 119-120 - Motor time (MT) of the rectus femoris was measured for seven normal subjects during rapid extension of the knee joint at $60^{\circ}$ flexion in the sitting position with and without external loads on the leg. MT prolonged linearly with external load from 0 to $4 \mathrm{~kg}$. Tangent of this linearity, the rate of tension development (RTD), was significantly different from one another of the subjects and was not related to MT without external load. RTD is a relevant index to assess an efficiency of rapid force generation of a muscle. - motor time ; external load

Motor time (MT) is defined as a latency from onset of EMG activities of a prime mover muscle to initiation of an actual movement in reaction time experiments. MT represents the time necessary for the muscle to develop the tension required for the initiation of rapid movement (Weiss 1965). MT has been regarded as an index of rapid force generation independent of the strength of maximal isometric voluntary contraction (MVC) (Irie et al. 1983). However, recent study suggests that MT is influenced by three independent factors, namely, the load including the weight of the body segment to be moved, the rate of tension development (RTD), and the tension-lag time (TLT) which is defined as a latency of tension measurable externally from onset of EMG activities. TLT is known to have little individual difference among normal subjects (Nakamura et al. 1984). MT prolongs in proportion to the increase in the weight to be moved (Nagasaki et al. 1983; Irie et al. 1983). RTD is obtained by calculating 'increment in weight loaded/increment in MT' in their studies. RTD should be interpreted as an efficiency of rapid force generation in an individual. The present study attempts to explore the influence of load and RTD on MT, measuring MTs of the rectus femoris for rapid knee extension under loading of different weights.

Seven normal men aged from 26 to 48 years participated in the experiment. By means of the water-immersion method, the mid-point of the left leg and foot was defined as the point corresponding to one half of the volume of them. The weight of the left leg and foot was roughly estimated as 7 per cent of the total body weight (Matsui 1956). The subject sat on a chair with his knees at $60^{\circ}$ flexion and with his heels supported by a horizontal bar. He was asked to extend his left knee as fast as possible in response to a tone stimulus. The details of measurement were already reported (Irie et al. 1983). In short, MT was measured as a time elapsed from onset of EMG activities of the rectus femoris to off-signal of a switch attached to the left heel. Five or more trials without external load were performed with intervals more than $10 \mathrm{sec}$. Then external loads of $1,2,3$ and $4 \mathrm{~kg}$ were attached to the mid-point of the left leg by belting a band with the lead, and the same trials were performed

Received September 25, 1984 ; accepted for publication November 12, 1984. 
for each weight. The means and s.D. of MTs were calculated from five trials in each weight of the load. Muscular tension produced by isometric maximal voluntary contraction (MVC) for left knee extension was also measured at the same position, using a strain gauge dynamometer (Irie et al. 1983).

The mean weight of the leg and foot was 4.82 (range $3.90-5.40) \mathrm{kg}$. The mean MT without external load (MTo) was 58.8 (48.4-78.0) msec, the mean RTD was $0.173(0.103-$ $0.385) \mathrm{kg} / \mathrm{msec}$, and the mean MVC was $18.78(13.23-27.97) \mathrm{kg} \cdot \mathrm{m}$. Analysis of covariance of regression on RTD was significant $(p<0.01)$, indicating RTD as a value characteristic to an individual. Correlation coefficients among the weight of the leg and foot, MTo, RTD and MVC were not significant except between the weight and MVC $(p<0.05)$. It is of worth to note that RTD was not related to MTo.

The present results suggest that RTD rather than MTo is a relevant measure to predict one's efficiency of rapid force generation. The relationship among TLT, RTD and load is depicted as MT $=\mathrm{TLT}+\mathrm{Load} / \mathrm{RTD}$. Thus, MTo of an individual does not reflect RTD due to individual difference of the weight of the body segment. RTD would reflect individual difference of neuromuscular function in two factors, namely, the neural command or firing rate of the nerve impulse, and the composition of various motor units in the muscle. RTD is a sensitive and easily measurable index to assess efficiency of rapid force generation of a muscle. RTD measurement would be applicable in such clinical fields as the analysis of pathophysiology and the evaluation of therapeutic effects in movement disorders.

\section{References}

1) Irie, N., Nagasaki, H. \& Nakamura, R. (1983) Motor reaction time as a measure of rapid force generation of a muscle. Tohoku J. exp. Med., 140, 145-151.

2) Matsui, S. (1956) Determination of center of gravity of human body in various postures. I. Center of gravity calculated with simplified mass values. Res. J. Educat., II, 65-76.

3) Nagasaki, H., Irie, N. \& Nakamura, R. (1983) Motor reaction time and stereotyped pattern of the EMG activities in ballistic elbow extension. Electromyogr. clin. Neurophysiol., 23, 167-181.

4) Nakamura, R., Nagasaki, H. \& Tsuji, I. (1984) Two components of motor time : tension lag and tension developing phase. Percept. Motor Skills, 59, 907-912.

5) Weiss, A.D. (1965) The locus of reaction time change with set, motivation, and age. J. Gerontol., 20, 60-64. 